\title{
Evaluation of repeat cytoreductive surgery and heated intraperitoneal chemotherapy for patients with recurrent peritoneal carcinomatosis from appendiceal and colorectal cancers: a multicentre Canadian study
}

\author{
Evan Jost, MD \\ Lloyd A. Mack, MD \\ Lucas Sideris, MD \\ Pierre Dube, MD, MSc \\ Walley Temple, MD \\ Antoine Bouchard-Fortier, \\ $\mathrm{MD}, \mathrm{MSc}$
}

Accepted June 27, 2019

\section{Correspondence to: \\ E. Jost \\ Foothills Medical Centre \\ 1403 29th St NW \\ Calgary AB T2N 2T9 \\ eajost@ucalgary.ca}

DOI: $10.1503 /$ cjs.002519
Background: Peritoneal recurrences after cytoreductive surgery (CRS) and heated intraperitoneal chemotherapy (HIPEC) for appendiceal and colorectal cancers are frequent. This study aimed to evaluate the safety, technical feasibility and perioperative and long-term outcomes of repeat CRS/HIPEC in patients with recurrent peritoneal carcinomatosis of colorectal and appendiceal origin.

Methods: Data were collected from patients treated from 2000 to 2016 for recurrent peritoneal carcinomatosis from appendiceal or colorectal cancer with CRS/HIPEC at 2 specialist centres. Data on demographics, procedure details, morbidity and survival were recorded. Analyses compared the iterations of CRS/HIPEC to assess the safety and effectiveness of repeat surgery.

Results: Of all patients who underwent CRS/HIPEC in the 2 centres, 37 patients underwent a repeat procedure. Operative time was similar for the first and second surgeries (412.1 v. $412.5 \mathrm{~min}, p=0.74$ ) but patients had a significantly lower peritoneal carcinoma index score with the second surgery (21.8 in the first iteration v. 9.53 in the second iteration, $p<0.001)$ and significantly less blood loss $(1762 \mathrm{~mL}$ in the first iteration v. $790 \mathrm{~mL}$ in the second iteration, $p=0.001)$. There was a nonsignificant decrease in grade III-IV complications and there was no 30-day mortality associated with repeat procedures. For patients with colorectal cancer, median disease-free survival was 9.6 months and median overall survival was 40 months. For patients with appendiceal cancer, median disease-free survival was 15 months and overall survival was 64.4 months.

Conclusion: Repeat CRS/HIPEC procedures for recurrent appendiceal and colorectal peritoneal carcinomatosis are safe in well-selected patients, without increased morbidity or mortality, and they are associated with significant long-term survival, particularly for patients with appendiceal cancers. These results support the use of repeat CRS/HIPEC in these patients.

Contexte : Les récurrences péritonéales après une chirurgie cytoréductrice (CCR) et une chimiothérapie hyperthermique intrapéritonéale (CHIP) pour les cancers de l'appendice et colorectaux sont fréquentes. Cette étude visait à évaluer l'innocuité, la faisabilité technique et les résultats périopératoires et à long terme d'une reprise de CCR/CHIP chez les patients qui présentent une récurrence de carcinomatose péritonéale ayant son origine au niveau colorectal ou de l'appendice.

Méthodes : Des données ont été recueillies sur des patients traités entre 2000 et 2016 pour une récurrence de carcinomatose péritonéale ayant son origine au niveau colorectal ou de l'appendice par CCR/CHIP dans 2 centres spécialisés. On a tenu compte des données démographiques, des détails des interventions, ainsi que de la morbidité et de la survie. Des analyses ont permis de comparer les premières et deuxièmes CCR/CHIP pour évaluer l'innocuité et l'efficacité des chirurgies répétées.

Résultats : De tous les patients soumis à des CCR/CHIP dans les 2 centres, 37 ont subi l'intervention de nouveau. Le temps opératoire a été similaire pour les premières et les deuxièmes chirurgies $(412,1$ c. $412,5 \mathrm{~min}, p=0,74)$, mais les patients présentaient un score de carcinomatose péritonéale beaucoup plus bas lors de la deuxième chirurgie $(21,8$ pour la première intervention c. 9,53 pour la seconde, $p<0,001)$ et des pertes sanguines significativement moindres $(1762 \mathrm{~mL}$ pour la première intervention c. $790 \mathrm{~mL}$ 
pour la seconde, $p=0,001)$. On a noté une diminution non significative des complications de grades III-IV et on n'a déploré aucune mortalité à 30 jours en lien avec la reprise de l'intervention. Pour les patients atteints d'un cancer colorectal, la survie médiane sans maladie a été de 9,6 mois et la survie médiane globale a été de 40 mois. Pour les patients atteints d'un cancer de l'appendice, la survie médiane sans maladie a été de 15 mois et la survie médiane globale a été de 64,4 mois.

Conclusion : La reprise des CCR/CHIP pour les récurrences de carcinomatose péritonéale ayant leur origine au niveau colorectal ou de l'appendice est sécuritaire chez les patients soigneusement sélectionnés, sans accroissement de la morbidité ou de la mortalité, et elles sont associées à une survie à long terme significative, particulièrement chez les patients ayant un cancer de l'appendice. Ces résultats appuient la reprise des CCR/CHIP chez ces patients.

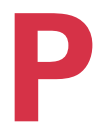

eritoneal carcinomatosis (PC) is defined by the presence of metastatic malignant deposits on the peritoneal surface that arise from a tumour exposed to the peritoneal cavity. It also includes tumours that arise and spread from the peritoneum itself. ${ }^{1}$

The incidence of $\mathrm{PC}$ varies with tumour type. For colorectal cancers, $5 \%-7 \%$ of patients are diagnosed with synchronous peritoneal metastases at the time of their initial cancer diagnosis. ${ }^{2}$ For appendiceal malignancies, the incidence is reported to be $1-3$ cases per million per year. ${ }^{3}$ The prognosis for patients with these tumours has traditionally been poor. ${ }^{4}$ However, treatment via cytoreductive surgery (CRS) and heated intraperitoneal chemotherapy (HIPEC) can result in 5-year survival rates of $40 \%$ and $90 \%$ in patients with colorectal and appendiceal primary tumours, respectively. ${ }^{5,6}$

Unfortunately, recurrence after CRS/HIPEC is very common. Recurrence after CRS/HIPEC has been reported in as many as $82 \%$ of patients with colorectal cancer after complete cytoreduction. ${ }^{7}$ For appendiceal cancers, this figure can approach $32 \% .{ }^{8}$ The most common site of recurrence following CRS/HIPEC in both colorectal and appendiceal cancers is the peritoneum. ${ }^{9}$ In our centres, patients are selected for repeat surgery on the basis of Eastern Cooperative Oncology Group (ECOG) status, absence of visceral or extra-abdominal metastases and disease extent.

Despite this high rate of recurrence, little is known about the perioperative outcomes and long-term survival benefits of repeat surgical treatment for isolated peritoneal recurrences following CRS/HIPEC. A systematic review on colorectal PC included studies reporting 3-year overall survival between $0 \%$ and $66 \%$, with reports of 3 -year disease-free survival of $14 \%-41.5 \% .^{7}$ A more recent systematic review of 91 patients reported a median survival of 20 months from the time of second CRS, with 2-year survival rates of up to $50 \% .{ }^{10} \mathrm{~A}$ study of an 18 -year experience with 3 or more iterations of CRS/HIPEC in patients with appendiceal malignancy reported a 5 -year overall survival rate of $70 \% .{ }^{11}$ This group also showed that survival was significantly affected by the completeness of cytoreduction, emphasizing the importance of patient selection. Another group reporting on 26 patients who underwent repeat
CRS/HIPEC for recurrent PC of appendiceal origin reported 5 -year survival rate of $35 \%$, with morbidity and mortality rates similar to those seen with the first iteration of CRS/HIPEC. ${ }^{5}$ There is wide variance in survival benefits and perioperative outcomes reported in the literature, as well as significant heterogeneity in the tumour types included in studies on repeat CRS/HIPEC. Further data are necessary to clarify these findings. Many of the studies on this topic present encouraging oncologic and safety results. We expect that adding another large, combined series of patients will add to the consensus regarding the feasibility and utility of repeating HIPEC/CRS after peritoneal recurrence.

The primary objective of our retrospective study was to evaluate the safety and technical feasibility of repeat CRS/HIPEC in patients with isolated peritoneal recurrence. Our secondary objective was to evaluate the effectiveness of this treatment modality with respect to survival and ultimate recurrence rate.

\section{Methods}

\section{Study design}

We conducted a multicentre, retrospective, cross-sectional study. We included consecutive patients with appendiceal or colorectal cancer presenting with recurrent $\mathrm{PC}$ treated with repeat CRS/HIPEC from February 2000 to March 2016 at the Foothills Medical Centre (Calgary, Alta.) and at the Maisonneuve-Rosemont Hospital (Montréal, Que.). Both centres are university-affiliated provincial tertiary care referral centres for the treatment of PC with CRS/HIPEC, each performing more than 60 procedures per year. All patients underwent repeat CRS/HIPEC at least twice during the study period. All patients had complete cytoreductions (completeness of cytoreduction [CCR] score 0 or 1). Patients were also included regardless of their peritoneal carcinomatosis index $(\mathrm{PCI})^{12}$ score at reoperation.

Patients were excluded if they did not undergo treatment with intraperitoneal chemotherapy. They were also excluded if they did not undergo cytoreductive surgery because their disease was deemed unresectable at the time of reoperation or if they underwent CRS but not HIPEC 
(e.g., patients who had CCR scores of 2 or more after an attempted CRS). Those who underwent CRS and HIPEC for malignancies other than appendiceal or colorectal cancer were also excluded. We did not require preoperative biopsy confirmation of recurrence, although many of the patients did have this at the time of surgery. The study was approved by the Conjoint Health Research Ethics Board at the University of Calgary and the Health Research Ethics Board at the University of Montreal.

\section{Data sources}

We queried the Synoptec/WebSMR data set in Calgary and the Montreal CRS/HIPEC database to identify an appropriate cohort of patients for this study. These data sets are prospectively maintained and capture $100 \%$ of CRS/HIPEC cases being done at these centres. Both incorporate extensive clinical data from all patients undergoing CRS/HIPEC operations. These databases were interrogated for patients who underwent repeat CRS/HIPEC surgeries within the study period. Data parameters collected included demographic parameters such as age and sex as well as tumour type and grade. We collected oncologic data such as neoadjuvant or adjuvant chemotherapy, as well as operative data such as type of intraperitoneal chemotherapy used, blood loss, transfusion requirements, operative time, PCI score preoperatively and CCR score. Finally, we collected outcome parameters such as grade III-IV complications as defined by the Clavien-Dindo classification, length of hospital stay, length of time to recurrence or death, and American Society of Anesthesiologists (ASA) score before repeat CRS/HIPEC. ${ }^{13,14}$ Data that were not available from the registries were collected by direct chart review.

\section{Operative details}

Patients in our centres who have recurrent PC must meet several criteria before being considered for repeat CRS and HIPEC. These criteria were previously published as guidelines from a pan-Canadian CRS/HIPEC working group. They include a good functional status (ECOG 0), age less than 65 years (but note that carefully selected older patients may be included), low-grade tumours, lack of evidence of extraperitoneal metastasis, and preoperative imaging suggesting that there is a possibility of achieving complete resection of gross tumour from the abdomen..$^{15,16}$ For patients with colorectal cancer, we typically consider that a PCI score over 20 is a relative contraindication to CRS/HIPEC. We also consider bowel obstruction or unresectable pelvic recurrences as contraindications to CRS/HIPEC. ${ }^{16}$ Patients with colorectal PC are, in general, treated with preoperative chemotherapy (typically FOLFOX or FOLFIRI regimens) to select those most likely to benefit from CRS/HIPEC.
Patients underwent CRS as initially described by Sugarbaker and colleagues. ${ }^{17}$ A laparotomy incision was created, followed by complete lysis of adhesions, and the PCI score was calculated preoperatively to assess the extent and size of tumour implants. One or more peritonectomy procedures were then undertaken to remove all visible disease, as well as electrofulguration of tumour nodules on the small bowel and visceral resections where necessary to remove primary tumours or sites of metastases.

Subsequently, a treatment of HIPEC was undertaken. For patients with all types of primary tumours, the most common chemotherapeutic agent used in Montreal was mitomycin $C$ at $10-12 \mathrm{mg} / \mathrm{m}^{2}$ in $2 \mathrm{~L} / \mathrm{m}^{2}$ of $0.9 \% \mathrm{NaCl}$. In Calgary, mitomycin $\mathrm{C}$ was given as $15 \mathrm{mg}$ diluted in $3 \mathrm{~L}$ of $5 \%$ dextrose in water (D5W) or Dianeal solution. Alternative agents were a combination of oxaliplatin $400 \mathrm{mg}$ given intraperitoneally in $2 \mathrm{~L} / \mathrm{m}^{2}$ of $0.9 \% \mathrm{NaCl}$ in Montreal or in $3 \mathrm{~L}$ of D5W or Dianeal solution in Calgary, and 5-fluorouracil $800 \mathrm{mg}$ given intravenously at both centres. In Montreal, the circulating time was 90 minutes for mitomycin C given intraperitoneally and 30 minutes for oxaliplatin given intraperitoneally, while in Calgary it was 60 minutes for both agents. In the second iteration of CRS/HIPEC, a different chemotherapeutic agent was used for the HIPEC than in the first iteration. These regimens varied between patients. The doses of chemotherapeutic agents used in the second iteration of surgery were not adjusted from those used for primary HIPEC procedures at each centre.

Any visceral anastomoses were then undertaken, and PCI and CCR scores were calculated at the conclusion of the procedure. CCR-0 was defined as no visible residual tumour, and CCR-1 was defined as tumour nodules no greater than $2.5 \mathrm{~mm}$ in size remaining. CCR-2 was defined as tumour nodules between $2.5 \mathrm{~mm}$ and $2.5 \mathrm{~cm}$ in size remaining, and CCR-3 was defined as tumour nodules greater than $2.5 \mathrm{~cm}$ remaining or the presence of confluent unresectable nodules. ${ }^{12}$ No HIPEC was undertaken when the CCR score was 2 or more, and these patients were not included in the final analysis.

\section{Statistical analysis}

We used descriptive statistics to evaluate patient and tumour characteristics. Means were compared using pairedsample Student $t$ tests, and categorical variables were compared using the related-sample $\mathrm{McNemar}$ test. We compared all operative and outcome measures between the first and subsequent iterations of CRS/HIPEC to determine if the repeat operations were associated with significantly worse outcomes. For these calculations, the first HIPEC/CRS was considered the index case and the operative parameters were compared between it and the second CRS/HIPEC. The operative parameters and outcome measures for each patient were compared with that patient's own variables from their initial surgery. Only 
2 patients underwent more than 2 CRS/HIPEC surgeries, and therefore these data were not included in the analysis. Primary outcome measures were time to recurrence or death, operative time, length of stay and complications. Secondary measures were blood loss, CCR score, preoperative ASA score and preoperative PCI score for initial and repeat CRS/HIPEC. A $p$ value $<0.05$ was defined as statistically significant. All data were analyzed using SPSS statistical software version 24.0.

\section{Results}

Forty-seven patients who were selected for more than 1 CRS/HIPEC during the study period. For 10 (21\%) of these patients a second CRS/HIPEC was attempted but their disease was found to be unresectable at the time of laparotomy; 37 patients had successful CRS/HIPEC. These 37 patients formed the study population. All of the patients who did not have a successful second CRS/HIPEC had appendiceal PC. For these patients, median overall survival was 42.1 months from the time of PC diagnosis. There was 1 postoperative complication among these patients following the patient's aborted second surgery, which was a pulmonary embolism. No patient died in the 30-day period following their second operation. Overall, 6 of 10 patients had died of their disease at the time of last follow-up.

Overall, approximately 1000 patients underwent primary CRS/HIPEC at the study institutions during the study period. The patients included in the study were predominantly female $(65 \%)$ with a mean age of 49 years at the first CRS/HIPEC (Table 1). Twenty-eight (76\%) of the patients had appendiceal primary tumours, with the majority (89\%) of the patients in the study overall having mucinous adenocarcinoma. The baseline characteristics of the appendiceal and colorectal cancer groups at each iteration of CRS/HIPEC are summarized in Table 2. Neoadjuvant chemotherapy was given to $57 \%$ of patients before the first CRS/HIPEC (CRS/HIPEC 1), with the most frequent regimens being based on FOLFIRI or FOLFOX. Nine $(32 \%)$ of the patients with appendiceal adenocarcinoma underwent neoadjuvant chemotherapy, versus $7(78 \%)$ of the patients with colorectal cancer. Before the second CRS/HIPEC, 7 (19\%) of the study patients underwent neoadjuvant chemotherapy, $5(71 \%)$ of whom had tumours of colorectal origin and 2 (29\%) of whom had tumours of appendiceal origin. FOLFOX- or FOLFIRI-based regimens were also used before HIPEC 2. However, there was no correlation between PCI score at CRS/HIPEC 2 (the second iteration of CRS/HIPEC) and whether a patient had neoadjuvant chemotherapy $(p=$ 0.19) (Table 1).

Results for intraoperative parameters are summarized in Table 3. The PCI score was lower in the second CRS/HIPEC than the first (9.53 v. $21.8, p<0.001)$. Operative time was similar for the first and second CRS/HIPEC, at 412.1 and 412.5 minutes, respectively $(p=0.74)$. Blood loss was significantly lower in the second surgery, with a mean of $790 \mathrm{~mL}$ versus $1762 \mathrm{~mL}$ for the first surgery $(p=$ 0.001). Blood transfusion requirements were also significantly lower in the second CRS/HIPEC, with means of 2.6 and 0.4 units in the first and second iterations, respectively $(p=0.002)$. The CCR score was similar between the operations. CCR-0 was achieved in $73 \%$ of cases in CRS/HIPEC 1 and in $87 \%$ of cases in CRS/HIPEC 2 ( $p=$ 0.23 ) (Table 3).

Data on immediate surgical outcomes are summarized in Table 4. There were no differences in complication rates between iterations of CRS/HIPEC. Following their first CRS/HIPEC, $46 \%$ of patients had a Clavien-Dindo class III or IV complication, and $43 \%$ had a complication following CRS/HIPEC $2(p=1.00)$ (Table 4). These

\begin{tabular}{|c|c|c|c|}
\hline Characteristic & $\begin{array}{c}\text { Patients with appendiceal } \\
\text { primary tumour } \\
n=28\end{array}$ & $\begin{array}{c}\text { Patients with colorectal } \\
\text { primary tumour } \\
n=9\end{array}$ & $p$ value \\
\hline Age, yr, mean & 49 & 47 & 0.63 \\
\hline Female sex, no. (\%) & $18(65)$ & $6(67)$ & 0.90 \\
\hline $\begin{array}{l}\text { Mean PCl score at second iteration of CRS/ } \\
\text { HIPEC }\end{array}$ & 28 & 8 & 0.034 \\
\hline Tumour grade, no. (\%) & & & 0.002 \\
\hline High & $3(11)$ & 7 (78) & \\
\hline Intermediate & $1(4)$ & 0 & \\
\hline Low & $23(82)$ & $2(22)$ & \\
\hline $\begin{array}{l}\text { ASA score before second iteration of CRS/HIPEC, } \\
\text { no. }(\%)\end{array}$ & & & 0.67 \\
\hline 1 & $10(36)$ & $4(44)$ & \\
\hline 2 & 11 (39) & $4(44)$ & \\
\hline 3 & $7(25)$ & $1(11)$ & \\
\hline
\end{tabular}


complications were most commonly intraabdominal abscesses requiring drainage or reoperation, but they also included thromboembolic disease, respiratory complications and ileus. Mean length of stay was shorter for CRS/HIPEC 2, (19.8 v. $28.4 \mathrm{~d}, p=0.06)$, although this result did not reach statistical significance (Table 4). There were no instances of 30-day mortality in either iteration of surgery.
Long-term survival and recurrence data are summarized in Table 5. Mean follow-up time was 63.5 months. For patients with appendiceal primary tumours, median diseasefree survival was nonsignificantly shorter following the second CRS/HIPEC (15.0 v. $17.7 \mathrm{mo}, p=0.75)$. For patients with colorectal primary tumours, there was also a nonsignificant trend to shorter disease-free survival after the second iteration of surgery ( 9.6 v. $21.6 \mathrm{mo}, p=0.11$ ). Peritoneal or

\begin{tabular}{|c|c|c|c|c|}
\hline \multirow[b]{2}{*}{ Characteristic } & \multicolumn{3}{|c|}{ Patients; iteration of CRS/HIPEC surgery } & \multirow[b]{2}{*}{$p$ value* } \\
\hline & First iteration & Second iteration & Third iteration & \\
\hline Appendiceal primary tumour, no. (\%) & $28(76)$ & $28(76)$ & $2(100)$ & NA \\
\hline Age, mean \pm SD & $49 \pm 11$ & $51 \pm 11$ & $56 \pm 7$ & NA \\
\hline ASA score, no. of patients & & & & NA \\
\hline Class 1 & 12 & 14 & 1 & \\
\hline Class 2 & 15 & 15 & 1 & \\
\hline Class 3 & 10 & 8 & 0 & \\
\hline Class 4 & 0 & 0 & 0 & \\
\hline Time between surgeries, $d$, mean $\pm S D$ & $932 \pm 562$ & $1391 \pm 1382$ & NA & 0.81 \\
\hline $\mathrm{PCl}$ score, mean $\pm \mathrm{SD}$ & $21.8 \pm 12.79$ & $9.53 \pm 7.26$ & $7.0 \pm 4.2$ & $<0.001$ \\
\hline \multicolumn{5}{|l|}{ HIPEC type, no. (\%) } \\
\hline Mitomycin C & $11(30)$ & $29(78)$ & $1(50)$ & NA \\
\hline Oxaliplatin & $10(27)$ & $1(3)$ & 0 & NA \\
\hline IP oxaliplatin + IV 5-FU & $16(43)$ & $7(19)$ & $1(50)$ & NA \\
\hline EPIC (1000 mg IP 5-FU ×5 days) & $10(27)$ & $7(19)$ & 0 & NA \\
\hline Neoadjuvant chemotherapy, no. (\%) & $16(43)$ & $7(19)$ & 0 & NA \\
\hline Adjuvant chemotherapy, no. (\%) & $12(32)$ & $3(8)$ & $1(50)$ & NA \\
\hline
\end{tabular}

Table 3. Intraoperative parameters for patients undergoing repeat CRS/HIPEC

\begin{tabular}{|c|c|c|c|c|}
\hline \multirow[b]{2}{*}{ Operative parameter } & \multicolumn{3}{|c|}{ Patients; iteration of CRS/HIPEC surgery } & \multirow[b]{2}{*}{$p$ value* } \\
\hline & First iteration & Second iteration & Third iteration & \\
\hline Operative time, min, mean $\pm S D$ & $412.1 \pm 135.7$ & $412.5 \pm 124.2$ & $375.0 \pm 21.2$ & 0.74 \\
\hline Intraoperative transfusions, units, mean \pm SD & $2.6 \pm 3.6$ & $0.4 \pm 0.81$ & $1.0 \pm 0.0$ & 0.002 \\
\hline Blood loss, $\mathrm{mL}$, mean $\pm \mathrm{SD}$ & $1762 \pm 1593$ & $790 \pm 502$ & $1000 \pm 0$ & 0.001 \\
\hline Completeness of cytoreduction score, no. (\%) & & & & 0.23 \\
\hline 0 & $27(73)$ & $32(87)$ & $2(100)$ & \\
\hline 1 & $10(27)$ & $5(14)$ & $0(0)$ & \\
\hline 2 & $0(0)$ & $0(0)$ & $0(0)$ & \\
\hline
\end{tabular}

Table 4. Early postoperative outcomes for patients undergoing repeat CRS/HIPEC

\begin{tabular}{|c|c|c|c|c|}
\hline \multirow[b]{2}{*}{ Outcome } & \multicolumn{3}{|c|}{ Patients; iteration of CRS/HIPEC surgery } & \multirow[b]{2}{*}{$p$ value } \\
\hline & First iteration & Second iteration & Third iteration & \\
\hline Complications, no. (\%) & $17(46)$ & $16(43)$ & $1(50)$ & 1.00 \\
\hline Length of stay, $d$, mean \pm SD & $28.4 \pm 19.5$ & $19.8 \pm 18.6$ & $11.5 \pm 0.71$ & 0.06 \\
\hline 30-d mortality & 0 & 0 & 0 & NA \\
\hline
\end{tabular}


distant disease was either persistent or recurrent in $46 \%$ of cases following the second CRS/HIPEC. Six patients $(16 \%)$ died during follow-up, of whom 3 had appendiceal primary tumours and 3 had colorectal primary tumours. Median overall survival for patients with appendiceal primary tumours and colorectal primary tumours was 64.4 months (standard deviation [SD] 38.7) and 40 months (SD 12.5), respectively (Table 5). Five-year overall survival rates for appendiceal cancers and colorectal cancers were $89 \%$ and $67 \%$, respectively (Fig. 1). At the time of last follow-up, $71 \%$ of patients with appendiceal cancer remained disease free and $100 \%$ of patients with colorectal cancer had had a recurrence (Fig. 2).

\section{Discussion}

Peritoneal carcinomatosis associated with colorectal or appendiceal malignancies is a relatively rare but potentially devastating pattern of metastasis. As survival from these tumours improves, the question of how best to treat recurrences of carcinomatosis without visceral metastases has arisen. One strategy that has been used is repeated CRS/HIPEC. The use of this technique is not widespread, and little is known about the perioperative and longer-term outcomes resulting from repeat CRS/HIPEC. The results of our study show that compared with the initial CRS/HIPEC surgery, repeat CRS/ HIPEC surgery is associated with similar operative time, decreased blood loss, decreased transfusion requirements and similar complication rates. With a mean follow-up of 63.5 months, tumour-related mortality was low, at $16 \%$.

A total of $54 \%$ of patients remained disease free at 5 years, of whom $71 \%$ had appendiceal primary tumours.
This proportion of patients with an appendiceal primary tumour is in line with both our own institutional series and the reported literature. The proportion of patients who underwent repeat CRS/HIPEC in our series was approximately $4 \%$. This is slightly lower than the value of $8 \%$ recently reported from another specialist centre. The difference may be a result of more conservative selection in our cases. ${ }^{18} \mathrm{~A}$ recently published series from our centre reported that the proportion of patients who underwent primary CRS/HIPEC with appendiceal tumours was $65.2 \%,{ }^{19}$ and a large 2019 series reported that the proportion of patients undergoing primary CRS/HIPEC with appendiceal tumours was $73 \%$. This is probably an effect of selection, as appendiceal PC tends to be more amenable to CRS/HIPEC as it typically has a more indolent course and is less invasive or infiltrative. This may also reflect the fact that the use of colorectal PC as an indication for CRS/HIPEC has been evolving over time, and CRS/HIPEC may not have been as frequently pursued for this indication at the earlier time points of our study. The baseline characteristics of the patients in each group differed, which points to the value of selection in these populations and the different biological behaviour of these tumours. The patients with appendiceal primary tumours had significantly higher PCI scores at their second HIPEC as well as a significantly higher proportion of low-grade tumours than the patients with colorectal primary tumours. Given that appendiceal PC is a more indolent disease, these patients may be offered repeat CRS/HIPEC even if they have more extensive disease. The higher proportion of high-grade tumours in the group with colorectal cancer may point to the fact that patients who have

Table 5. Long-term outcomes following repeat CRS/HIPEC for patients with recurrent peritoneal carcinomatosis

\begin{tabular}{|c|c|c|c|c|c|c|}
\hline Outcome & \multicolumn{4}{|c|}{ Patients; iteration of CRS/HIPEC surgery } & \multicolumn{2}{|c|}{$p$ value } \\
\hline \multicolumn{7}{|c|}{ Disease-free survival, mo, median \pm SD } \\
\hline Appendix & $17.7 \pm 12.8$ & $15.0 \pm 8.3$ & $9.8 \pm 5.9$ & & 0.75 & \\
\hline Colon & $21.6 \pm 10.1$ & $9.6 \pm 5.7$ & & & 0.11 & \\
\hline Colon & $9(100)$ & $9(100)$ & & & & \\
\hline \multicolumn{7}{|l|}{$\begin{array}{l}\text { Overall survival, mo, } \\
\text { median } \pm \mathrm{SD}\end{array}$} \\
\hline Appendix & & & & $64 \pm 39$ & & \\
\hline Colon & & & & $40 \pm 12$ & & \\
\hline \multicolumn{7}{|c|}{$\begin{array}{l}\text { Overall survival by CCR score and primary } \\
\text { tumour type, mo, median } \pm \mathrm{SD}\end{array}$} \\
\hline CCR-1 colon & & & & $45 \pm 7$ & & \\
\hline
\end{tabular}


histologically aggressive tumours are more likely to have recurrences of PC. ${ }^{20}$

An analysis of the intraoperative parameters suggests that the second CRS/HIPEC compares favourably with the first CRS/HIPEC with respect to the safety and difficulty of the operation in well-selected patients. Operative times and CCR scores were similar for the 2 iterations. It is possible that the lower burden of PC in the second iteration of surgery allowed a similarly complete cytoreduction to be performed. ${ }^{21}$ A study comparing patients with high-volume PC of appendiceal origin to those with low-volume PC reported median operating times that were 26 minutes shorter for low-volume disease (PCI score < 12). ${ }^{21}$ Intraoperative blood loss and transfusion requirements were significantly lower in the second CRS/HIPEC. Immediate postoperative outcomes were similar between the 2 iterations of surgery. Complication rates were almost identical, which would indicate that the safety of repeat CRS/HIPEC was similar to that of the initial surgery. Unfortunately, $10(21 \%)$ patients were found to have unresectable disease at the time of attempted repeat CRS/HIPEC. Patients who underwent unsuccesful repeat procedures certainly would have experienced increased pain and would have had to recover from surgery, which are undesirable outcomes, particularly in a palliative population. The postoperative pulmonary embolism experienced by 1 of the patients was another adverse outcome. In addition, these patients had lower median overall survival than the patients who

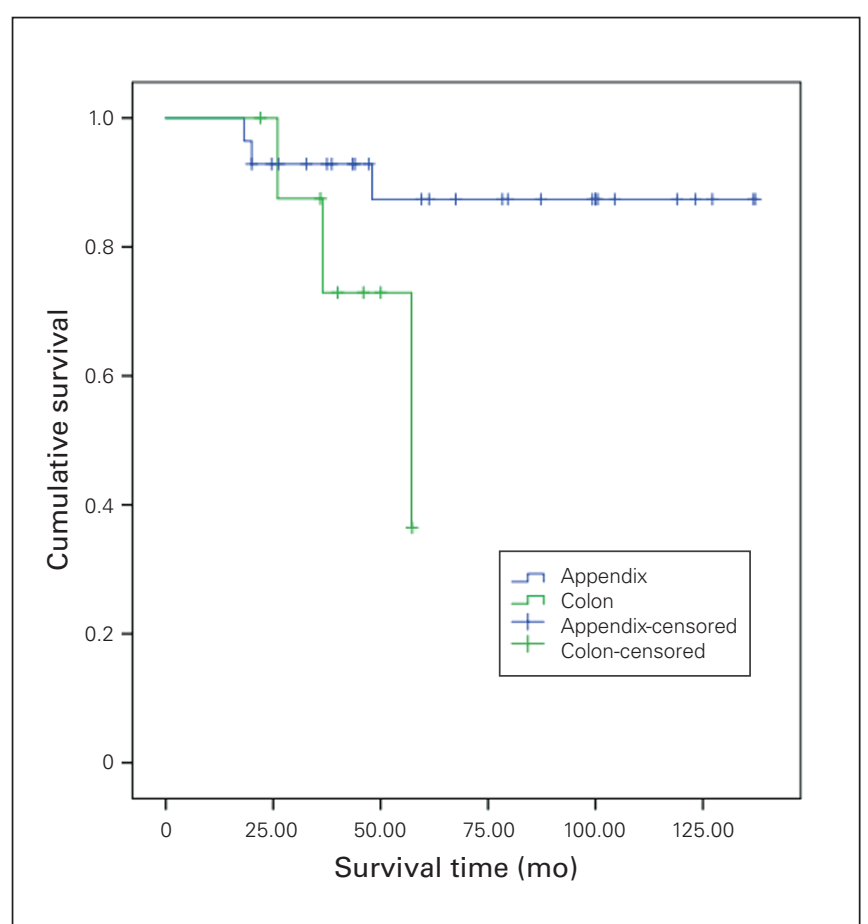

Fig. 1. Overall survival from first CRS/HIPEC by primary tumour site for patients undergoing repeat CRS/HIPEC surgery with complete cytoreduction. Censored = cases censored by date of death from any cause; CRS = cytoreductive surgery; HIPEC = heated intraperitoneal chemotherapy. underwent successful CRS/HIPEC. This may indicate that the surgery had a beneficial effect for the patients in whom it was succesful or that the patients in whom the surgery was unsuccessful had worse tumour biology. The rate of aborted CRS may drop as the sensitivity of noninvasive imaging modalities continues to improve.

There was a trend toward decreased disease-free survival following the second CRS/HIPEC for both patients with appendiceal primary tumours and those with colorectal primary tumours: median disease-free survival was 2.7 months shorter in patients with appendiceal cancer and 12 months shorter in patients with colorectal cancer. However, neither observation was statistically significant. Other studies echo these results, with most describing shorter or similar disease-free survival following a second iteration of CRS/HIPEC. ${ }^{5,7,10,11}$ A recent systematic review of 190 patients with recurrent carcinomatosis of colorectal origin who underwent secondary CRS reported diseasefree survival for the first CRS/HIPEC of up to 23 months, with the median disease-free survival for the second iteration being 13.7 months. ${ }^{7}$ Klaver and colleagues, in a study including patients with colorectal and appendiceal PC, reported a median disease-free survival of 4.5 months following repeat CRS/HIPEC (range 1-22 mo). ${ }^{22}$ In the largest study to date of repeat CRS/HIPEC for patients with appendiceal primary tumours, Yan and colleagues reported in 2007 a 5 -year progression-free survival rate of $75 \%,{ }^{23}$ while Sardi and colleagues in 2013 reported a median

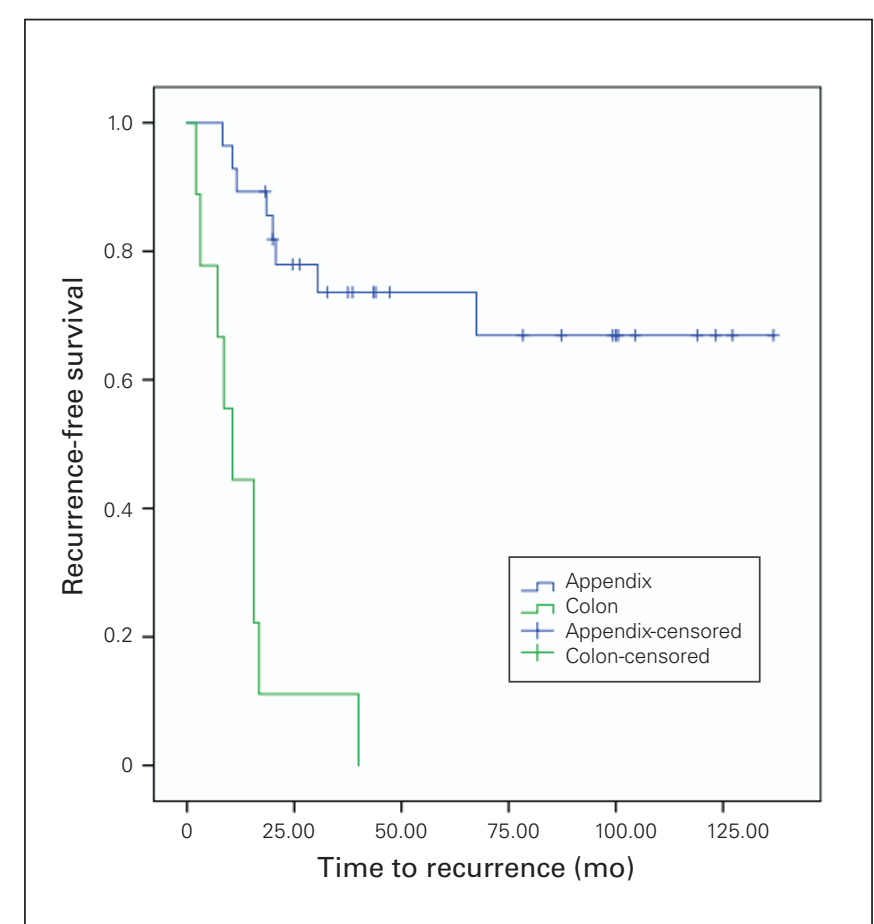

Fig. 2. Recurrence-free survival by tumour type for patients following repeat CRS/HIPEC surgery with a complete cytoreduction. Censored $=$ cases censored by date of first documented recurrence on imaging or clinical documentation; CRS = cytoreductive surgery; HIPEC = heated intraperitoneal chemotherapy. 
disease-free survival of 33.9 months $^{5}$ in the most recently published series to report disease-free survival. Our results correspond well with those of the other groups, given that all of the patients with colorectal cancer had experienced a recurrence at 63 months, whereas only $25 \%$ of patients with appendiceal malignancies had experienced a recurrence over the same follow-up period. Interestingly, the Peritoneal Malignancy Institute in Basingstoke, United Kingdom, reported their 5-year median disease-free survival rate to be $74.9 \%$ for the primary CRS/HIPEC surgery in a series of 1000 patients with appendiceal malignancies. ${ }^{24}$ Our results for the second iteration of CRS/HIPEC are in line with this observation, suggesting that the second iteration of CRS/HIPEC may return patients to their original prognosis.

The finding of a median overall survival of 64 months for patients with appendiceal primary tumours and 40 months for patients with colorectal primary tumours from the first CRS/HIPEC and a 5-year overall survival rate of $89 \%$ for patients with appendiceal cancers and $67 \%$ for patients with colorectal cancers compares favourably with the findings of other groups, with median overall survival being reported as between 18 and 55.7 months for patients with colon and appendiceal malignancies, respectively. ${ }^{7}$ A recent series from a French group reported a 5 -year overall survival rate of $45.8 \%$ in patients who received an iterative curative-intent procedure after recurrence, compared with $4.7 \%$ in patients who did not. ${ }^{25}$ Our results provide further confirmation that intervention is likely to result in significant benefit for patients whose prognoses would otherwise be dismal. The recurrence rate of $100 \%$ for colorectal cancer and $29 \%$ for appendiceal cancer is also in accordance with the findings of other studies, with reports ranging from $22.5 \%$ to $82 \%{ }^{26,27}$ for patients with colorectal primary tumours, and from $45 \%$ to $54 \%{ }^{6,23}$ for patients with appendiceal primary tumours.

These results are in the context of recently presented data from a randomized study that call into question the oncologic effectiveness of intraperitoneal chemotherapy when added to cytoreductive surgery. This study reported that there was no difference in overall survival or recurrence-free survival for patients who had HIPEC versus patients who did not, although it is notable that patients in both arms of this study underwent CRS and that the survival in both arms was exceptionally high, with median overall survival of over 41 months in both groups. ${ }^{28}$ This study may certainly inform future treatment of PC. However, the HIPEC regimen undertaken in this study is not the same as that used in our centre, and it is unclear whether a different intraperitoneal agent may yield better results. Furthermore, this study reports significantly better overall survival and recurrence-free survival for patients with intermediate PCI scores, suggesting that selected patients may benefit from HIPEC in addition to CRS. There were few additional complications reported with the addition of
HIPEC, a finding that we have also observed in our own centres. Taken together, we feel that these findings by themselves do not justify the omission of HIPEC from our protocol, and we do believe that patients may derive benefit from intraperitoneal chemotherapy in addition to cytoreductive surgery.

It is also possible that the encouraging median overall survival in our study and other series is related to the increasing efficacy of systemic chemotherapy. Advances in systemic therapies for cancers of colorectal and other origins are progressing at an impressive rate. However, a recent meta-analysis performed on 10553 patients with colorectal PC from 14 randomized trials described a median overall survival of 17.1 months in patients given combination chemotherapy and targeted therapy. ${ }^{29}$ The median overall survival for patients who received chemotherapy alone was 16.3 months. None of the patients in this study received CRS or HIPEC for their disease. These survival times are dramatically shorter than those seen for CRS/HIPEC in our study. This result from a very large patient sample suggests that patients may derive some benefit from CRS/HIPEC.

Our findings indicate that repeat CRS/HIPEC is technically feasible and appropriate in well-selected patients. Our study is from 2 institutions that perform a high volume of these surgeries (approximately 1000 between the 2 centres during the study period). Additional strengths of our study are that the surgeries were performed by only 5 surgeons during the study period and that the second surgery was almost always performed by the same surgeon as the first. This means that our data are very consistent in nature. Our study is also larger than many studies on this topic, given that the average number of patients in prior studies ranged from 2 to 125 . $^{7,18}$

\section{Limitations}

A limitation of this study is its retrospective nature and the fact that HIPEC chemotherapy and perfusion duration were not standardized between the study sites. However, we do not believe that this had a significant impact as patients were selected for repeat procedures according to similar criteria at the 2 sites. In addition, although this is a relatively large patient series for this type of surgery, the numbers of patients are small, limiting the statistical certainty of our conclusions. These patients were highly selected, representing the healthiest patients with the best possible tumour biology. The study is also limited by the lack of a control group.

\section{Conclusion}

The results of this study show that repeat CRS/HIPEC surgery is safe, effective and technically feasible in wellselected patients. Compared with the initial CRS/HIPEC, we achieved similar complication rates, as well as lower 
operative times and intraoperative blood loss in the second iteration of surgery. We also achieved long-term disease-free and overall survival for patients with a second recurrence of PC, particularly in the population with appendiceal cancer. We feel that this indicates that patients who have evidence of isolated peritoneal recurrence after CRS/HIPEC surgery should be considered for possible repeat surgery in a tertiary centre.

Affiliations: From the Department of Surgery, Foothills Medical Centre, University of Calgary, Calgary, Alta. (Jost, Mack, Temple, BouchardFortier); and the Department of Surgery, Maisonneuve-Rosemont Hospital, Université de Montréal, Montréal, Que. (Sideris, Dube).

\section{Competing interests: None declared.}

Contributors: All authors designed the study. E. Jost, P. Dube and A. Bouchard-Fortier acquired the data, which E. Jost, L. Mack, L. Sideris and A. Bouchard-Fortier analyzed. E. Jost wrote the article, which all authors reviewed and approved for publication.

\section{References}

1. Verwaal VJ, Boot H, Aleman BM, et al. Recurrences after peritoneal carcinomatosis of colorectal origin treated by cytoreduction and hyperthermic intraperitoneal chemotherapy: location, treatment, and outcome. Ann Surg Oncol 2004;11:375-9.

2. Segelman J, Granath F, Holm T, et al. Incidence, prevalence, and risk factors for peritoneal carcinomatosis from colorectal cancer. $\mathrm{Br} \mathcal{F}$ Surg 2012;99:699-705.

3. Son IT, Ahn S, Park KJ, et al. Comparison of long-term oncological outcomes of appendiceal cancer and colon cancer: a multicenter retrospective study. Surg Oncol 2016;25:37-43.

4. Chan CH, Cusack JC, Ryan DP. A critical look at local-regional management of peritoneal metastasis. Hematol Oncol Clin North Am 2015;29:153-8

5. Sardi A, Jimenez WA, Nieroda C, et al. Repeated cytoreductive surgery and hyperthermic intraperitoneal chemotherapy in peritoneal carcinomatosis from appendiceal cancer: analysis of survival outcomes. Eur 7 Surg Oncol 2013;39:1207-13.

6. Brouquet A, Goere D, Lefevre JH, et al. The second procedure combining complete cytoreductive surgery and intraperitoneal chemotherapy for isolated peritoneal recurrence: postoperative course and long-term outcome. Ann Surg Oncol 2009;16:2744-51.

7. van Oudheusden TR, Nienhuijs SW, Luyer MD, et al. Incidence and treatment of recurrent disease after cytoreductive surgery and intraperitoneal chemotherapy for peritoneally metastasized colorectal cancer: a systematic review. Eur 7 Surg Oncol 2015;41:1269-77.

8. Ihemelandu C, Fernandez S, Sugarbaker PH. A prognostic model for predicting overall survival in patients with peritoneal surface malignancy of an appendiceal origin treated with cytoreductive surgery and hyperthermic intraperitoneal chemotherapy. Ann Surg Oncol 2017;24:2266-72.

9. Braam HJ, van Oudheusden TR, de Hingh IH, et al. Patterns of recurrence following complete cytoreductive surgery and hyperthermic intraperitoneal chemotherapy in patients with peritoneal carcinomatosis of colorectal cancer. F Surg Oncol 2014;109:841-7.

10. Williams BHM, Alzahrani NA, Chan DL, et al. Repeat cytoreductive surgery (CRS) for recurrent colorectal peritoneal metastases: yes or no? Eur 7 Surg Oncol 2014;40:943-9.

11. Mohamed F, Chang D, Sugarbaker PH. Third look surgery and beyond for appendiceal malignancy with peritoneal dissemination. $\mathcal{F}$ Surg Oncol 2003;83:5-12, discussion 12-3.
12. Gilly FN, Cotte E, Brigand C, et al. Quantitative prognostic indices in peritoneal carcinomatosis. Eur 7 Surg Oncol 2006;32:597-601.

13. Dindo D, Demartines N, Clavien PA. Classification of surgical complications: a new proposal with evaluation in a cohort of 6336 patients and results of a survey. Ann Surg 2004;240:205-13.

14. Dripps RD, Lamont A, Eckenhoff JE. The role of anesthesia in surgical mortality. FAMA 1961;178:261-6.

15. Oken MM, Creech RH, Tormey DC, et al. Toxicity and response criteria of the Eastern Cooperative Oncology Group. Am 7 Clin Oncol 1982;5:649-55.

16. Dube P, Sideris L, Law C, et al. Guidelines on the use of cytoreductive surgery and hyperthermic intraperitoneal chemotherapy in patients with peritoneal surface malignancy arising from colorectal or appendiceal neoplasms. Curr Oncol 2015;22:e100-12.

17. Sugarbaker PH. Surgical management of carcinomatosis from colorectal cancer. Clin Colon Rectal Surg 2005;18:190-203.

18. Choudhry HA, Bednar F, Shuai Y, et al. Repeat cytoreductive surgery-hyperthermic intraperitoneal chemoperfusion is feasible and offers survival benefit in select patients with peritoneal metastases. Ann Surg Oncol 2019;26:1445-53.

19. Hamilton T, Lanuke K, Mack LA, et al. Long-term followup in the treatment of peritoneal carcinomatosis. Am F Surg 2011;201:650-4.

20. Winer J, Zenati M, Ramalingam L, et al. Impact of aggressive histology and location of primary tumour on the efficacy of surgical therapy for peritoneal carcinomatosis of colorectal origin. Ann Surg Oncol 2014;21:1456-62.

21. Polanco PM, Ding Y, Knox JM, et al. Outcomes of cytoreductive surgery and hyperthermic intraperitoneal chemoperfusion in patients with high-grade, high-volume disseminated mucinous neoplasms. Ann Surg Oncol 2016;23:382-90.

22. Klaver YL, Chua TC, Verwaal VJ, et al. Secondary cytoreductive surgery and peri-operative intraperitoneal chemotherapy for peritoneal recurrence of colorectal and appendiceal peritoneal carcinomatosis following prior primary cytoreduction. F Surg Oncol 2013; 107:585-90.

23. Yan TD, Bijelic L, Sugarbaker PH. Critical analysis of treatment failure after complete cytoreductive surgery and perioperative intraperitoneal chemotherapy for peritoneal dissemination from appendiceal mucinous neoplasms. Ann Surg Oncol 2007;14:2289-99.

24. Ansari N, Chandrakumaran K, Dayal S, et al. Cytoreductive surgery and hyperthermic intraperitoneal chemotherapy in 1000 patients with perforated appendiceal epithelial tumours. Eur 7 Surg Oncol 2016;42:1035-41.

25. Gelli M, Huguenin JFL, de Baere T, et al. Peritoneal and extraperitoneal relapse after previous curative treatment of peritoneal metastases from colorectal cancer: What survival can we expect? Eur 7 Surg Oncol 2018;100:94-103.

26. Goere D, Malka D, Tzanis D, et al. Is there a possibility of a cure in patients with colorectal peritoneal carcinomatosis amenable to complete cytoreductive surgery and intraperitoneal chemotherapy? Ann Surg 2013;257:1065-71.

27. Cashin PH, Graf W, Nygren P, et al. Cytoreductive surgery and intraperitoneal chemotherapy for colorectal peritoneal carcinomatosis: prognosis and treatment of recurrences in a cohort study. Eur $\mathcal{F}$ Surg Oncol 2012;38:509-15.

28. Quenet F, Elias D, Roca L, et al. A unicancer phase III trial of hyperthermic intra-peritoneal chemotherapy (HIPEC) for colorectal peritoneal carcinomatosis, PRODIGE 7. Eur 7 Surg Oncol 2019;45:e22.

29. Franko J, Shi Q, Meyers JP, et al. Prognosis of patients with peritoneal metastatic colorectal cancer given systemic therapy: an analysis of individual patient data from prospective randomised trials from the analysis and research in cancers of the digestive system (ARCAD) database. Lancet Oncol 2016;17:1709-19. 